\title{
Treatment Strategy for Post-Hepatectomy Recurrent Hepatocellular Carcinoma: A Single Center Experience with 556 Consecutive Cases in China
}

\author{
Jing Li1,2*, Liang Huang2*, Caifeng Liu'², Maixuan Qiu², Jianjun Yan², Shaohua Wei1, Yiqun Yan ${ }^{2}$ \\ ${ }^{1}$ Department of General Surgery, The Second Affiliated Hospital of Soochow University, Suzhou, China \\ ${ }^{2}$ Department of Hepatic Surgery, Shanghai Eastern Hepatobiliary Surgery Hospital, Shanghai, China \\ Email: ehbhyyq@163.com, shaohuawei2010@sina.com
}

How to cite this paper: Li, J., Huang, L., Liu, C.F., Qiu, M.X., Yan, J.J., Wei, S.H. and Yan, Y.Q. (2020) Treatment Strategy for Post-Hepatectomy Recurrent Hepatocellular Carcinoma: A Single Center Experience with 556 Consecutive Cases in China. Journal of Cancer Therapy, 11, 389-400. https://doi.org/10.4236/jct.2020.117034

Received: May 20, 2020

Accepted: July 12, 2020

Published: July 15, 2020

Copyright $\odot 2020$ by author(s) and Scientific Research Publishing Inc. This work is licensed under the Creative Commons Attribution International License (CC BY 4.0).

http://creativecommons.org/licenses/by/4.0/

(c) (i) Open Access

\begin{abstract}
Background: Treatment strategy for recurrent hepatocellular carcinoma (HCC) remains scantily defined. This study was aimed to establish a treatment strategy to manage post-hepatectomy recurrent HCC and report the clinical outcomes. Methods: From January 2006 to December 2016, 556 consecutive patients who developed post-hepatectomy HCC recurrence were enrolled in the study. The patients were clinically stratified and treated according to a strategy established by a multi-disciplinary team. Clinical data and survival times were collected prospectively and analyzed retrospectively. Results: According to the strategy, there were 298 (53.6\%), 214 (38.5\%), 32 (5.7\%) and $12(2.2 \%)$ patients stratified into Early, Intermediate, Advanced and Terminal stages, respectively. In Early stage patients, 164 (55.0\%) received curative treatment in the form of repeat resection or local ablation, 134 (45.0\%) received transarterial chemoembolization (TACE), and the 1-, 3-, and 5 -year overall survival (OS) rates were $82.0 \%, 46.8 \%$ and $37.3 \%$, respectively. In Intermediate stage patients, 207 (96.7\%) received TACE, 7 (3.3\%) radiotherapy, and the 1-, 3-, and 5-year OS rates were $73.2 \%, 31.8 \%$ and $15.9 \%$, respectively. In Advanced stage patients, 22 patients received sorafenib, 10 radiotherapy, and the mean survival time (MST) was $25.1 \pm 3.1$ months. All the 12 patients in Terminal stage received the best supportive treatment, and the MST was $6.5 \pm$ 3.4 months. Clinical stages and duration of disease-free interval were independent factors relating to overall survival. Conclusions: A treatment strategy derived from the Barcelona Clinic Liver Cancer staging system, with some modifications, has been successfully established to manage post-hepatectomy recurrent $\mathrm{HCC}$, and the clinical outcomes were commendable.
\end{abstract}




\section{Keywords}

Post-Hepatectomy Recurrent Hepatocellular Carcinoma, Clinical Classification, Treatment Strategy, Clinical Outcomes

\section{Introduction}

Despite continuous advances in the diagnosis and treatment of hepatocellular carcinoma (HCC), tumor recurrence after liver resection with curative-intent is still common [1]. Our previously reported tumor recurrence rate for HCC was $86.2 \%$ at 5 years after liver resection [2]. A study focusing on the treatment of HCC recurrence and its related prognostic factors on long-term survival is becoming crucially important in the overall management of HCC patients.

Of the many options which are available to treat recurrent HCC, including repeat resection (RR), local ablation therapy (LAT), transarterial chemoembolization (TACE), radiotherapy, targeted drug therapy, and salvage liver transplantation (SLT), there are only a few reports which compared the effectiveness of these different treatment modalities [3] [4] [5] [6]. The treatment strategy for post-hepatectomy HCC recurrence remains poorly defined. The European Association for the Study of the Liver (EASL) guidelines recommend that treatment should be based on the Barcelona Clinic Liver Cancer (BCLC) staging and treatment system [7].

However, recommendations based on the BCLC staging and treatment system in treating primary HCC may not be simply copied to treat post-hepatectomy recurrent HCC. Not only the recurrent tumor data, the patient's present hepatic functional reserve and the basic characteristics, but also the surgical and pathologic profiles of the previously resected tumor need to be considered in the decision making process on the treatment of HCC recurrence [8]. Therefore, a multi-disciplinary team (MDT) approach is much more suitable for managing patients with recurrent HCC [9]. With the recommendation of the MDT, this study established a modified strategic algorithm derived from the BCLC staging system and presented the long-term survival outcomes of a large consecutive cohort of patients who developed post-hepatectomy recurrent HCC.

\section{Methods}

\subsection{Patients}

Consecutive patients who underwent liver resection at the Eastern Hepatobiliary Surgery Hospital from January 2006 to December 2014 were followed-up regularly after surgery. At each follow-up visit, a detailed history taking and physical examination were carried out. Serum alpha-fetoprotein (AFP), liver function tests and abdominal ultrasound were done once every 2 months, and contrast-enhanced computed tomography (CT) or magnetic resonance imaging 
(MRI) was performed at least once every 6 months, or earlier when clinically indicated. When extrahepatic recurrence was suspected, positron emission tomography-computed tomography (PET-CT) was conducted. HCC recurrence was diagnosed based on clinical and radiological findings using the same criteria as in diagnosing primary HCC, as recommended by EASL guidelines using imaging +/- tumor markers [7]. Once the diagnosis of recurrent HCC was made, patients were prospectively enrolled in the study.

This study was approved by the Ethics Committee of the Eastern Hepatobiliary Hospital and it followed the standards of the Declaration of Helsinki. Informed consent was obtained from all patients for their data to be used for research.

\subsection{Clinical Classification of Post-Hepatectomy Recurrent HCC}

According to the MDT, patients with post-hepatectomy recurrent HCC were stratified into four stages (Early, Intermediate, Advanced and Terminal) (Figure 1). The patients at Early stage were those who had the BCLC stage 0 and stage A recurrent tumors and without satellitosis or microvascular invasion (MVI) in the pathological findings obtained from the initial hepatectomy. Accordingly, the patients with satellitosis or MVI at the initial hepatectomy were classified into the Intermediate stage, together with those who had BCLC stage B recurrent tumors. Advanced stage and Terminal stage classification were equal to the BCLC stage $\mathrm{C}$ and stage $\mathrm{D}$, respectively.

\subsection{Treatment Strategy for Post-Hepatectomy Recurrent HCC}

Patients with Early stage of recurrent HCC would be recommended to receive RR, LAT or TACE. Due to a donor shortage and financial issues, SLT was seldom recommended for patients and thus not included in this study. RR was strongly recommended by the MDT to treat Early stage of recurrent HCC if technically feasible. However, the indications of the second surgery were very different from that of the first one and much stricter. Briefly, the extra conditions that applied to the candidates for RR, in addition to BCLC criteria, were as follow: 1) without satellite lesions or MVI associated with the primary tumor; 2) up to 3 lesions in the relatively easily removed location in the liver and no one neighboring the primary operative area, especially if close to major intrahepatic tracts; 3 ) $<3$ segment resections involved in the first surgery. LAT was performed for patients with tumor size $<3 \mathrm{~cm}$ if technically feasible to puncture percutaneously. Those patients who were not suitable for RR or LAT would receive TACE.

For patients with Intermediate stage of recurrent HCC, TACE was the first line treatment option, while very few patients would receive radiotherapy due to very poor arterial infusion of the tumors. For patients with Advanced stage of recurrent HCC, targeted therapy with sorafenib was the main choice, while those with solitary metastasis in the lung or bone would receive radiotherapy. Finally, patients fitting into the Terminal stage would receive only the best supportive 
treatment (Figure 1).

Open repeat hepatectomy was performed using the clamp-crushing method with inflow-blood-occlusion. LAT included radiofrequency ablation (RFA), microwave ablation (MWA) and percutaneous ethanol injection (PEI). TACE was performed by injection of chemotherapeutic agents using doxorubicin and cisplatin mixed with lipiodol followed by embolization of tumor feeding arteries with gelatin sponge particles. Stereotactic body radiation therapy (SBRT) was used for radiotherapy.

\subsection{Follow-Up}

All patients with HCC recurrence were followed-up in the outpatient clinic. Physical examination followed by liver function and tumor marker levels was routinely carried out once every month. CT/MRI or PET-CT was performed when clinically indicated. Further treatment was recommended by the MDT for tumor recurrence or for inadequate control of the initial recurrent disease.

\subsection{Statistical Analysis}

The analysis was performed using the SPSS 18 software program (Chicago, IL). Continuous variables were presented as mean \pm standard deviation (SD) or median (range), and categorical variables as numbers (percentage). Overall survival (OS) was considered from the date of recurrence until the last available follow-up

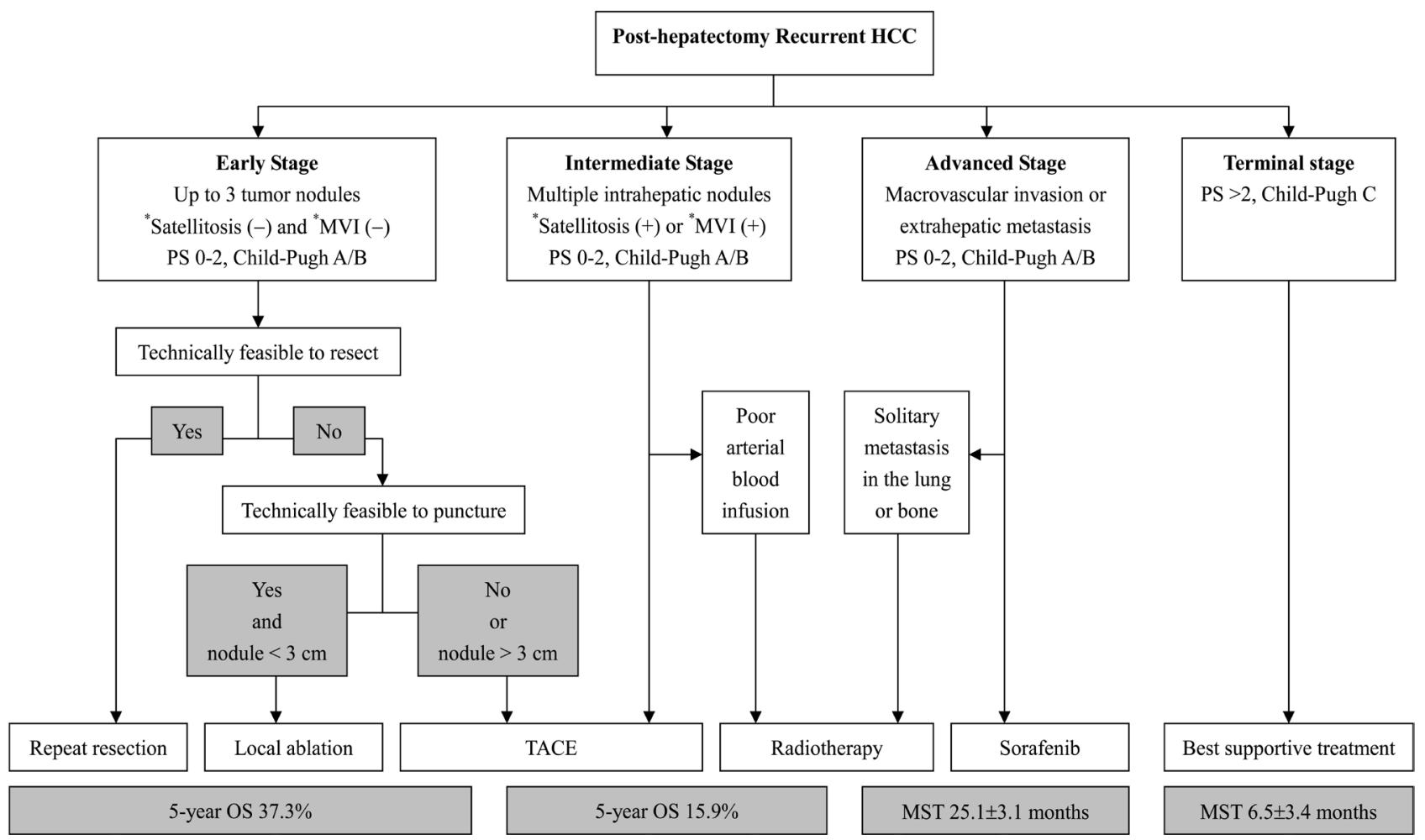

*at initial resection. HCC: hepatocellular carcinoma; MVI: microvascular invasion; PS: performance status; TACE: transarterial chemoembolization; OS: overall survival; MST: mean survival time.

Figure 1. Treatment strategy for post-hepatectomy recurrent HCC. 
or death. Survival rates were analyzed using the Kaplan-Meier method, and group differences were compared using the log-rank test. Prognostic factors for overall survival were identified with the COX proportional hazards regression model. A P value $<0.05$ was considered statistically significant.

\section{Results}

\subsection{Patients}

On the closing date of 31 December 2016, 556 patients who were diagnosed to suffer from recurrent HCC were enrolled in the study. There were 448 males and 108 females, with a mean age of $53.4 \pm 11.7$ years. The median time between initial HCC resection and recurrence was 8 (range 2 to 195) months. At the time when HCC recurrence was diagnosed, 516 (92.8\%), 17 (3.1\%) and 23 (4.1\%) patients had developed intrahepatic, extrahepatic and both intrahepatic and extrahepatic recurrences respectively. Based on the clinical classification of our strategy, there were 298 (53.6\%), 214 (38.5\%), 32 (5.7\%) and $12(2.2 \%)$ patients belonging to the Early, Intermediate, Advanced and Terminal stages, respectively. The clinical features of these 556 patients at the time of recurrence are shown in Table 1.

\subsection{Treatment of Recurrence}

Among the 556 patients with post-hepatectomy recurrent HCC, 341 (61.3\%) patients received TACE, 90 (16.2\%) RR, 74 (13.3\%) LAT, 17 (3.1\%) radiotherapy and $34(6.1 \%)$ palliative treatment.

In patients with Early stage recurrent HCC, 164 (55.0\%) received curative treatment in the form of RR or LAT, while $134(45.0 \%)$ received TACE. In patients with Intermediate stage recurrent HCC, 207 (96.7\%) received TACE, and $7(3.3 \%)$ radiotherapy due to very poor arterial blood infusion of the tumors. In patients with Advanced stage recurrent HCC, sorafenib tablets were prescribed for 22 patients, and radiotherapy was given to 10 patients who had a solitary metastatic tumor in the lung or bone. All the 12 patients with Terminal stage disease received best supportive treatment.

\subsection{Survival Outcomes and Prognostic Factors}

After a median follow-up of 31 months (range: 10 - 176 months), 392 (70.5\%) patients with post-hepatectomy recurrent HCC had died. The 1-, 3-, and 5-year OS rates of the entire cohort after recurrence were $72.2 \%, 34.3 \%$, and $25.0 \%$, respectively. Table 2 summarizes the survival outcomes of patients in relation to variables. Especially, the 1-, 3-, and 5-year OS rates after recurrence for patients in the Early stage were $82.0 \%, 46.8 \%$ and $37.3 \%$, respectively, whereas in the Intermediate stage, they were $73.2 \%, 31.8 \%$ and $15.9 \%$, respectively. The mean survival time after recurrence for patients in the Advanced and Terminal stages were $25.1 \pm 3.1$ months and $6.5 \pm 3.4$ months, respectively. Survival was related to the clinical stages (Figure 2). Patients who received curative treatments in the 
form of RR and LAT achieved better survival outcomes compared with those who received TACE $(\mathrm{P}<0.001)$. The survival outcomes between patients who received RR or LAT showed no significant difference $(P=0.848)$ (Figure 3 ).

Table 1. Clinical features of the 556 patients with recurrent HCC.

\begin{tabular}{|c|c|c|}
\hline Variable & $\mathbf{N}$ & Percent (\%) \\
\hline Age (ys) & \multicolumn{2}{|c|}{$53.4 \pm 11.7$} \\
\hline$<60$ & 441 & 79.3 \\
\hline$\geq 60$ & 115 & 20.7 \\
\hline \multicolumn{3}{|l|}{ Sex } \\
\hline Male & 448 & 80.6 \\
\hline Female & 108 & 19.4 \\
\hline \multicolumn{3}{|l|}{ Hepatitis etiology } \\
\hline HBV & 513 & 92.3 \\
\hline $\mathrm{HCV}$ & 2 & 0.3 \\
\hline No & 41 & 7.4 \\
\hline Serum AFP level (ng/ml) & \multicolumn{2}{|c|}{$130.6(0.7-339,567)$} \\
\hline$<20 \mathrm{ng} / \mathrm{ml}$ & 189 & 34.0 \\
\hline$\geq 20 \mathrm{ng} / \mathrm{ml}$ & 367 & 66.0 \\
\hline \multicolumn{3}{|l|}{ Liver function } \\
\hline Child-pugh A & 473 & 85.1 \\
\hline Child-pugh B & 68 & 12.2 \\
\hline Child-pugh C & 15 & 2.7 \\
\hline \multicolumn{3}{|l|}{ Site of recurrence } \\
\hline Intrahepatic & 516 & 92.8 \\
\hline Extrahepatic & 17 & 3.1 \\
\hline Intrahepatic and extrahepatic & 23 & 4.1 \\
\hline Tumor size $(\mathrm{cm})$ & \multicolumn{2}{|c|}{$6.1 \pm 10.5$} \\
\hline \multicolumn{3}{|l|}{ Tumor nodule } \\
\hline Single & 298 & 51.7 \\
\hline Multiple & 258 & 44.8 \\
\hline \multicolumn{3}{|l|}{ Tumor thrombus } \\
\hline Yes & 40 & 6.9 \\
\hline No & 516 & 89.6 \\
\hline \multicolumn{3}{|l|}{ Clinical stages } \\
\hline Early stage & 298 & 53.6 \\
\hline Intermediate stage & 214 & 38.5 \\
\hline Advanced stage & 32 & 5.7 \\
\hline Terminal stage & 12 & 2.2 \\
\hline Disease-free interval (months) & \multicolumn{2}{|c|}{$8(2-195)$} \\
\hline
\end{tabular}

HCC: hepatocellular carcinoma; HBV: hepatitis B virus; HCV: hepatitis C virus; AFP: alpha-fetoprotein; BCLC: barcelona clinic liver cancer. 
Table 2. Survival outcomes for recurrent HCC.

\begin{tabular}{|c|c|c|c|c|c|}
\hline Variable & $\begin{array}{l}\text { Mean survival } \\
\text { time (months) }\end{array}$ & 1-year OS & 3-year OS & 5 -year OS & $P$ value \\
\hline Site of recurrence & & & & & $<0.001$ \\
\hline Intrahepatic & $56.1 \pm 3.3$ & $73.4 \%$ & $37.0 \%$ & $27.2 \%$ & \\
\hline Extrahepatic & $24.2 \pm 3.8$ & $68.6 \%$ & $20.6 \%$ & 0 & \\
\hline Intrahepatic and extrahepatic & $20.0 \pm 4.4$ & $57.1 \%$ & $3.3 \%$ & 0 & \\
\hline Tumor nodules & & & & & $<0.001$ \\
\hline Single & $72.3 \pm 4.8$ & $81.8 \%$ & $46.5 \%$ & $39.2 \%$ & \\
\hline Mutiple & $31.2 \pm 2.9$ & $61.3 \%$ & $20.9 \%$ & $10.1 \%$ & \\
\hline Tumor thrombus & & & & & $<0.001$ \\
\hline Yes & $18.0 \pm 4.7$ & $40.0 \%$ & $10.0 \%$ & $5.0 \%$ & \\
\hline No & $55.6 \pm 3.2$ & $74.7 \%$ & $36.3 \%$ & $26.7 \%$ & \\
\hline Clinical stages & & & & & $<0.001$ \\
\hline Early stage & $70.7 \pm 4.7$ & $82.0 \%$ & $46.8 \%$ & $37.3 \%$ & \\
\hline Intermediate stage & $42.8 \pm 5.7$ & $73.2 \%$ & $31.8 \%$ & $15.9 \%$ & \\
\hline Advanced stage & $25.1 \pm 3.1$ & $55.5 \%$ & $14.2 \%$ & 0 & \\
\hline Terminal stage & $6.5 \pm 3.4$ & $10.0 \%$ & 0 & 0 & \\
\hline Treatment strategies & & & & & $<0.001$ \\
\hline $\mathrm{RR}$ & $73.0 \pm 8.1$ & $94.0 \%$ & $48.5 \%$ & $38.4 \%$ & \\
\hline LAT & $83.1 \pm 9.3$ & $84.7 \%$ & $52.0 \%$ & $16.7 \%$ & \\
\hline TACE & $47.3 \pm 3.6$ & $67.3 \%$ & $30.1 \%$ & $21.2 \%$ & \\
\hline Radiotherapy & $27.0 \pm 7.0$ & $64.7 \%$ & $11.8 \%$ & 0 & \\
\hline Palliative treatment & $11.3 \pm 2.2$ & $34.4 \%$ & $3.1 \%$ & 0 & \\
\hline Disease-free interval & & & & & $<0.001$ \\
\hline$\leq 1$ year & $37.9 \pm 3.0$ & $60.5 \%$ & $23.9 \%$ & $16.1 \%$ & \\
\hline$>1$ years & $73.6 \pm 9.2$ & $91.8 \%$ & $52.9 \%$ & $39.5 \%$ & \\
\hline
\end{tabular}

HCC: hepatocellular carcinoma; OS: overall survival; BCLC: Barcelona Clinic Liver Cancer; RR: repeat resection; LAT: local ablation therapy; TACE: transarterial chemoembolization.

Six variables including site of recurrence, tumor nodules, tumor thrombus, treatment options, clinical stages and disease-free interval were selected on multivariate analysis using the Cox regression model, and the results showed that two independent factors relating to survival after recurrence were identified: early stages of recurrent HCC was associated with better survival $(\mathrm{HR}=1.804$, $95 \% \mathrm{CI}$ : $0.923-3.186, \mathrm{P}=0.018$ ), while early HCC recurrence of $\leq 1$ year predicted a poorer survival $(\mathrm{HR}=0.610,95 \% \mathrm{CI}$ : $0.453-0.822, \mathrm{P}=0.001)$ (Table 3$)$.

\section{Discussion}

The EASL recommends to reassess patients with HCC recurrence with the BCLC staging system and to retreat them accordingly [7], but no data is available to 


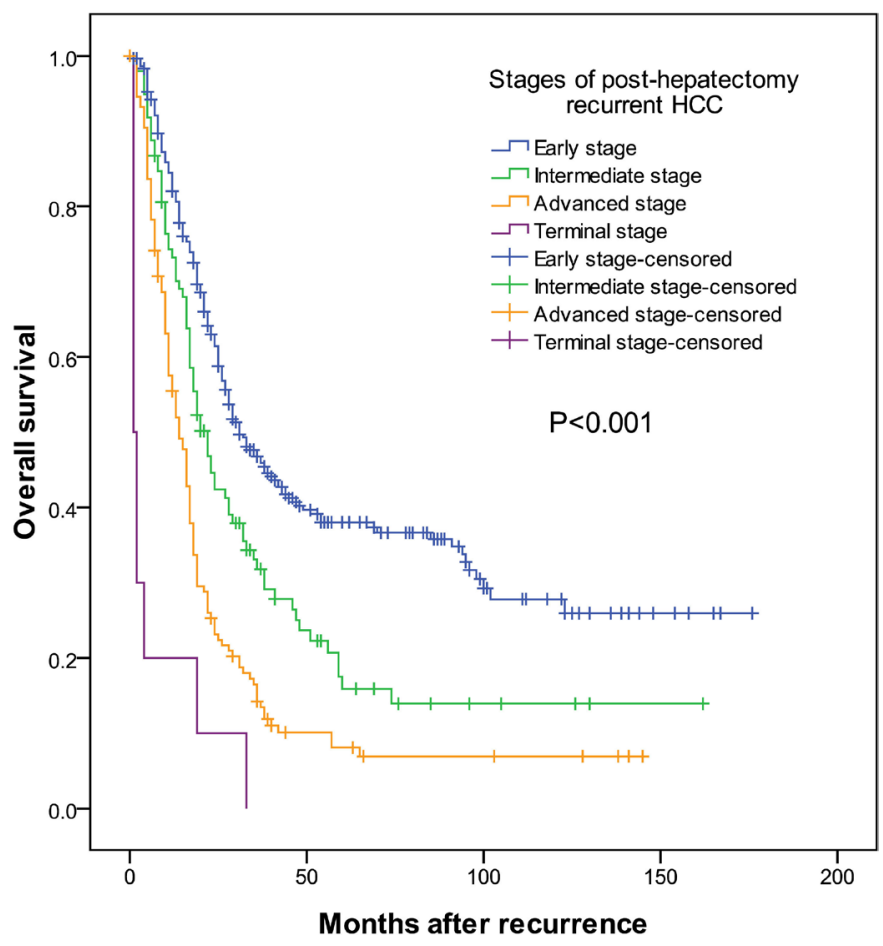

HCC: hepatocellular carcinoma.

Figure 2. Overall survival of patients with different clinical stages of post-hepatectomy recurrent HCC.

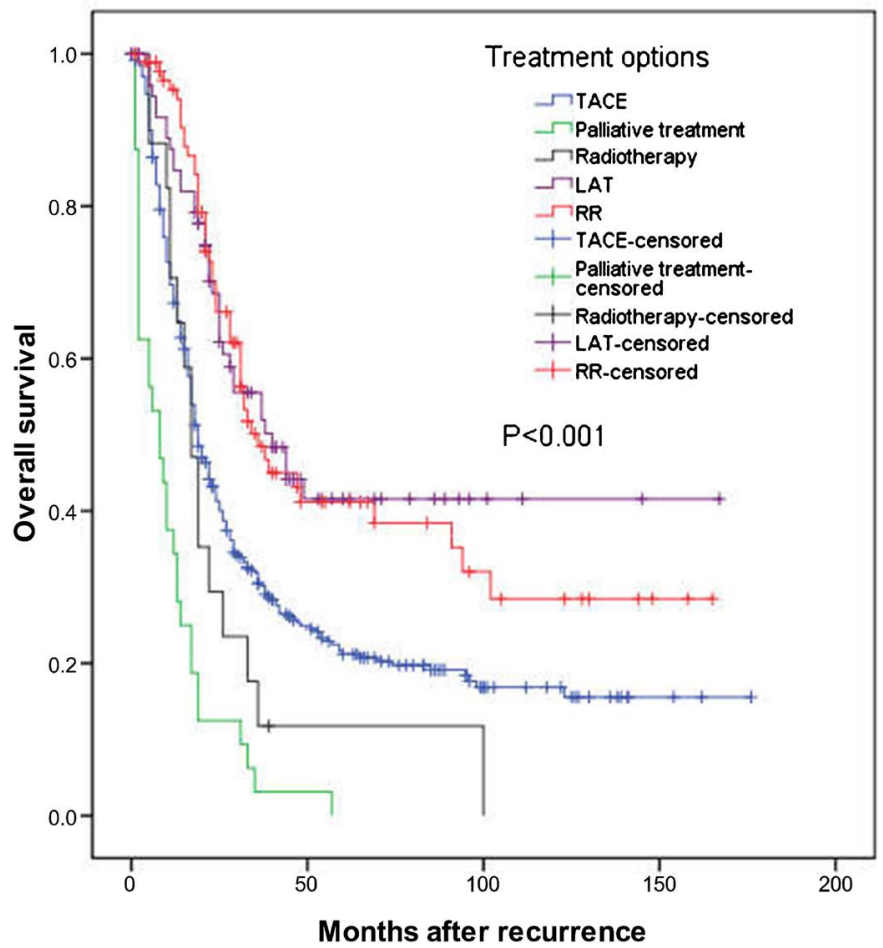

TACE: transarterial chemoembolization; LAT: local ablation therapy; RR: repeat resection; HCC: hepatocellular carcinoma.

Figure 3. Survival curves of patients with post-hepatectomy recurrent HCC undergoing different treatments. 
Table 3. Prognostic factors of survival on multivariate analysis using the Cox regression model in patients with recurrent HCC.

\begin{tabular}{cccc}
\hline Variable & HR & $95 \%$ CI & P value \\
\hline Site of recurrence (intrahepatic vs others) & 1.306 & $0.685-2.487$ & 0.715 \\
Tumor nodules (multiple vs single) & 0.743 & $0.543-1.018$ & 0.065 \\
Tumor thrombus (yes vs no) & 0.748 & $0.488-1.148$ & 0.184 \\
Treatment options (RR vs others) & 1.183 & $0.837-1.671$ & 0.075 \\
Clinical stages (early stage vs others) & 1.804 & $0.923-3.186$ & 0.018 \\
Disease-free interval ( $\leq 1$ year vs $>1$ year) & 0.610 & $0.453-0.822$ & 0.001
\end{tabular}

HCC: hepatocellular carcinoma; HR: hazard ratio; CI: confidence interval; RR: repeat resection; BCLC: barcelona clinic liver cancer.

support this recommendation. Assessment of post-hepatectomy recurrent HCC, in contrast to that of primary HCC, should also focus on the histological features of the primary resected tumor and the potential impact of these histologic features on the results of the treatment of recurrent disease [8]. Also, the impact of the previous surgery needs to be considered if local interventions such as RR and LAT are to be introduced. In our clinical center, we have accumulated extensive experience in managing patients with recurrent HCC. Since 2001, we have applied the MDT approach to treat recurrent HCC, and thereafter we established a strategy to manage patients with post-hepatectomy HCC in 2006. This strategy derived from the BCLC staging system but some modifications were made. Up to now, we have applied this strategy to manage our patients for more than 10 years, and the long-term survival outcomes of patients after recurrence are commendable (Figure 1).

In this study, as previously mentioned, patients with post-hepatectomy recurrent HCC were stratified into four stages (Early, Intermediate, Advanced and Terminal), with two extra factors (satellitosis and MVI that the primary resected tumor presented) involved when staging the recurrent tumors. It has been widely acknowledged that patients who present with satellitosis or MVI are more likely to develop early recurrence after curative resection and thus have poor long-term survival [10] [11] [12]. In order to avoid the likelihood of further short-term recurrence, we excluded those patients from the early stage, although whose recurrent tumors had BCLC stage 0 and stage A specifications, presumably to receive RR or LAT in BCLC staging system and, stratified them into the intermediate stage. The results showed that the long-term survival outcomes of the four stages as plotted by the Kaplan-Meier curves separated nicely (Figure 2). Moreover, on multivariate analysis, the clinical stages were identified to be one of the independent factors related to overall survival.

Treatment options for post-hepatectomy recurrent HCC were different from that of primary HCC. RR and LAT, as the curative therapy, have been reported to achieve the best long-term survival rate for HCC recurrence in well-selected 
patients [1] [8] [13] [14] [15] [16]. However, according to our treatment strategy, only $55 \%$ of patients in the early stage were candidates for RR and LAT. Contraindications of surgery include multiple recurrences, inadequate volume of future liver remnant, unfavourable location of recurrence, and extensive adhesions after the initial liver resection. In the patients with normal serum AFP, a confident diagnosis of a HCC nodule $<1 \mathrm{~cm}$ is currently difficult if not impossible [17]. Given an uncertainty in diagnosing the number of recurrent tumor nodules, treatment choice could always be difficult to make, therefore, TACE could be used as a starting treatment as well as to detect small liver nodules which were not shown on CT and MRI. Further treatments could then be carried out depending on, by means of follow-up CT scan findings, whether those nodules had iodine accumulations. Thus it was not surprising that TACE became a frequent measure on up to $45 \%$ of the early stage patients in this study. In addition, radiotherapy was also introduced into the treatment strategy as it has become a considerable choice of treatment in selected patients with poor arterial infusion of tumor, and for patients with extrahepatic metastasis, especially bony metastasis[6] [18] [19].

Previous studies have shown that tumor size, tumor number, macrovascular invasion, degree of liver dysfunction, presence of portal hypertension and the duration of disease-free interval were independent factors related to the overall survival of patients with recurrent HCC [13] [14] [15] [16]. These, however, were all retrospective studies on patients who were selected for curative treatment. The present study was a prospective study with a large consecutive series of patients. The results showed that two independent factors, the clinical stages of recurrent HCC and the duration of the disease-free interval after the initial liver resection, were related to the overall survival of post-hepatectomy HCC recurrence. These meaningful findings may provide a guide to manage patients with post-hepatectomy recurrent HCC.

A major limitation of this study was the unavailability of salvage liver transplantation (SLT) for this cohort of patients. Previous studies have shown that in selected patients SLT can achieve similar 5-year OS and better disease-free survival compared with RR and RFA [5] [20]. In China, very few patients with recurrent HCC have the chance to undergo SLT due to the severe shortage of donor livers and financial issues.

\section{Conclusion}

In conclusion, this study has tried to establish a treatment strategy with some differences from the BCLC staging system to manage patients with post-hepatectomy recurrent HCC. Patients were stratified into four stages (Early, Intermediate, Advanced and Terminal stage), and the long-term survival outcomes of the four stages as plotted by the Kaplan-Meier curves separated nicely. The clinical stages of recurrent HCC and the length of the post-hepatectomy disease-free interval were independent factors related to overall survival. 


\section{Acknowledgements}

This work was supported by the Youth Research Found Project of Jiading District Commission of Health and Family Planning (No. 2017-QN-02).

\section{Conflicts of Interest}

The authors declare no conflicts of interest regarding the publication of this paper.

\section{References}

[1] Yagi, R., Midorikawa, Y., Moriguchi, M., et al. (2018) Liver Resection for Recurrent Hepatocellular Carcinoma to Improve Survivability: A Proposal of Indication Criteria. Surgery, 163, 1250-1256. https://doi.org/10.1016/j.surg.2017.12.022

[2] Li, J., Huang, L., Yan, J.J., Qiu, M.X. and Yan, Y.Q. (2018) Liver Resection for Hepatocellular Carcinoma: Personal Experiences in a Series of 1330 Consecutive Cases in China. ANZ Journal of Surgery, 88, E713-717. https://doi.org/10.1111/ans.14381

[3] Roayaie, S., Bassi, D., Tarehi, P., Labow, D. and Schwartz, M. (2011) Second Hepatic Resection for Recurrent Hepatocellular Cancer: A Western Experience. Journal of Hepatology, 55, 346-350. https://doi.org/10.1016/j.jhep.2010.11.026

[4] Soog, K.D., Lee, M.W., Rhim, H., et al. (2018) Percutaneous US/MRI Fusion-Guided Radiofrequency Ablation for Recurrent Subcentimeter Hepatocellular Carcinoma: Technical Feasibility and Therapeutic Outcomes. Radiology, 288, 878-886. https://doi.org/10.1148/radiol.2018172743

[5] Lim, C., Shinkawa, H., Hasegawa, K., et al. (2017) Salvage Liver Transplantation or Repeat Hepatectomy for Recurrent Hepatocellular Carcinoma: An Intent-to-Treat Analysis. Liver Transplantation, 23, 1553-1563. https://doi.org/10.1002/lt.24952

[6] Su, T.S., Liang, P., Lu, H.Z., et al. (2016) Stereotactic Body Radiation Therapy for Small Primary or Recurrent Hepatocellular Carcinoma in 132 Chinese Patients. Journal of Surgical Oncology, 113, 181-187. https://doi.org/10.1002/jso.24128

[7] European Association for the Study of the Liver (2012) European Organisation for Research and Treatment of Cancer. EASLEORTC Clinical Practice Guidelines: Management of Hepatocellular Carcinoma. Journal of Hepatology, 56, 908-943. https://doi.org/10.1016/j.jhep.2011.12.001

[8] Meniconi, R.L., Komatsu, S., Perdigao, F., Boelle, P.Y., Soubrane, O. and Scatton, O. (2015) Recurrent Hepatocellular Carcinoma: A Western Strategy That Emphasizes the Impact of Pathologic Profile of the First Resection. Surgery, 157, 454-462. https://doi.org/10.1016/j.surg.2014.10.011

[9] Guy, J., Kelley, R.K., Roberts, J., Kerlan, R., Yao, F. and Terrault, N. (2012) Multidisciplinary Management of Hepatocellular Carcinoma. Clinical Gastroenterology and Hepatology, 10, 354-362. https://doi.org/10.1016/j.cgh.2011.11.008

[10] Huang, Z.Y., Liang, B.Y., Xiong, M., et al. (2012) Long-Term Outcomes of Repeat Hepatic Resection in Patients with Recurrent Hepatocellular Carcinoma and Analysis of Recurrent Types and Their Prognosis: A Single-Center Experience in China. Annals of Surgical Oncology, 19, 2515-2525. https://doi.org/10.1245/s10434-012-2269-7

[11] Du, M., Chen, L., Zhao, J., et al. (2014) Microvascular Invasion (MVI) Is a Poorer Prognostic Predictor for Small Hepatocellular Carcinoma. BMC Cancer, 14, 38. 
https://doi.org/10.1186/1471-2407-14-38

[12] Feng, L.H., Dong, H., Lau, W.Y., et al. (2017) Novel Microvascular Invasion Based Prognostic Nomograms to Predict Survival Outcomes in Patients after R0 Resection for Hepatocellular Carcinoma. Journal of Cancer Research and Clinical Oncology, 143, 293-303. https://doi.org/10.1007/s00432-016-2286-1

[13] Li, M., Wang, Z., Cao, J., et al. (2019) Risk Factors and Prognosis of Patients with Recurrent Hepatocellular Carcinoma Who Undergo Liver Re-Resections. European Journal of Surgical Oncology, 45, 1684-1690. https://doi.org/10.1016/j.ejso.2019.04.008

[14] Chan, A.C., Poon, R.T., Cheung, T.T., et al. (2012) Survival Analysis of Re-Resection versus Radiofrequency Ablation for Intrahepatic Recurrence after Hepatectomy for Hepatocellular Carcinoma. World Journal of Surgery, 36, 151-156. https://doi.org/10.1007/s00268-011-1323-0

[15] Guo, W.X., Zhai, B., Lai, E.C.H., et al. (2008) Percutaneous Radiofrequency Ablation versus Partial Hepatectomy for Multicentric Small Hepatocellular Carcinomas: A Nonrandomized Comparative Study. World Journal of Surgery, 15, 3484-3493.

[16] Song, K.D., Lim, H.K., Rhim, H., et al. (2015) Repeated Hepatic Resection versus Radiofrequency Ablation for Recurrent Hepatocellular Carcinoma after Hepatic Resection: A Propensity Score Matching Study. Radiology, 275, 599-608. https://doi.org/10.1148/radiol.14141568

[17] Bruix, J. and Sherman, M. (2011) Management of Hepatocellular Carcinoma: An Update. Hepatology, 53, 1020-1022. https://doi.org/10.1002/hep.24199

[18] Kim, D.Y., Park, J.W., Kim, T.H., et al. (2017) Risk-Adapted Simultaneous Integrated Boost-Proton Beam Therapy (SIB-PBT) for Advanced Hepatocellular Carcinoma with Tumour Vascular Thrombosis. Radiotherapy and Oncology, 122, 122-129. https://doi.org/10.1016/j.radonc.2016.12.014

[19] Kim, T.H., Park, J.W., Kim, B.H., et al. (2018) Optimal Time of Tumour Response Evaluation and Effectiveness of Hypofractionated Proton Beam Therapy for Inoperable or Recurrent Hepatocellular Carcinoma. Oncotarget, 9, 4034-4043. https://doi.org/10.18632/oncotarget.23428

[20] Zhang, X., Li, C., Wen, T., Peng, W. and Yang, J. (2017) Treatment for Intrahepatic Recurrence after Curative Resection of Hepatocellular Carcinoma: Salvage Liver Transplantation or Re-Resection/Radiofrequency Ablation? A Retrospective Cohort Study. International Journal of Surgery, 46, 178-185.

https://doi.org/10.1016/j.ijsu.2017.09.001 